\title{
Evaluation of client and staff satisfaction with a Goal Planning project implemented with people with spinal cord injuries
}

\author{
GM MacLeod $^{1}$ and L Macleod ${ }^{2}$ \\ ${ }^{1}$ Clinical Psychologist, Queen Elizabeth National Spinal Injuries Centre, Southern General Hospital, Glasgow G51, \\ Scotland; ${ }^{2}$ Trainee Clinical Psychologist, Department of Psychological Medicine, University of Glasgow, Gartnavel \\ Royal Hospital, Glasgow G12, Scotland
}

\begin{abstract}
This study assesses client satisfaction and canvasses staff opinions concerning the first year of a Goal Planning rehabilitation programme implemented with in-patients who had sustained a severe spinal cord injury. Questionnaires were completed by 33 patients. As a keyworker system is an integral part of Goal Planning, separate questionnaires were completed by 13 staff/keyworkers. The results indicated that patients believed Goal Planning to be informative about their injury and helpful in promoting a sense of control over their rehabilitation. In addition, a positive, and statistically significant association was found between perceived informativeness and control and the implications for successful rehabilitation are considered. Staff also gave favourable feedback regarding the perceived benefits of Goal Planning for patients, relatives and staff, although they acknowledged the resulting increase in their workload.
\end{abstract}

Keywords: spinal cord injury (SCI); rehabilitation; control

\section{Introduction}

The British Government publications The Health of the Nation ${ }^{1}$ and The Patients' Charter ${ }^{2}$ both stipulate the importance of fully involving and informing patients about their treatment, In the area of rehabilitation, increasing patient participation and control has been linked to favourable outcomes across a wide range of health care settings. ${ }^{3}$ Specifically amongst people with spinal cord injuries, Norris-Baker et al ${ }^{4}$ have shown in-patient involvement in rehabilitation to be the best predictor of medical and behavioural outcome status. In particular, these authors found that patients who participate in active rehabilitation are more likely to be independent after discharge from hospital and less likely to be readmitted with medical complications.

Based on such findings, Kennedy et $a l^{5,6}$ have developed 'Goal Planning': a needs driven approach with the aim being 'to empower people with a spinal cord injury to preserve, develop and reintegrate quality life goals, through appropriate scientifically-based interventions, a needs-determined service and responsive staff support'. (p. 17). With an emphasis on involving patients in decision making and minimising institutionalisation and dependency, Goal Planning focuses not only on the physical aspects of a person's injury, but on a wide range of skills necessary for reintegration into the community. They point out that 'these necessary skills will only be acquired if individuals understand the rehabilitation process, are

Correspondence: GM MacLeod fully involved in decisions about this process, agree key goals, and play an active part in their rehabilitation' (p. 17).

The Goal Planning process begins with a detailed needs assessment and then uses a behavioural change strategy originally developed by Houts and $\operatorname{Scott}^{7}$ for working with people with learning disabilities. This approach is characterised by a recognition of the importance of client involvement and an emphasis on needs and strengths rather than weaknesses and disabilities. It attempts to specify goals in clear behavioural terms and set realistic targets while recognising the importance of professional input from a trained multi-disciplinary team. Goal Planning operates on the premise that providing patients with information about their condition and their rehabilitation, and promoting autonomy in choice and decision making, facilitates effective coping and thus facilitates rehabilitation. Kennedy et $a l^{5}$ have shown that where a Goal Planning approach has been formally adopted with spinally injured patients, they spend more time in therapy areas during the day, less time in solitary activity and more time in verbal interaction. Thus Goal Planning may be an effective way of maximising the therapeutic potential of the rehabilitation environment.

This study attempts to assess client satisfaction in the first year of a Goal Planning programme which was used to direct the rehabilitation of patients with spinal cord injuries admitted to the Queen Elizabeth National Spinal Injuries Centre (QUENSIC) in 
Glasgow. Although there are perhaps methodological limitations and theoretical difficulties in interpreting data from customer feedback, Trinder ${ }^{8}$ points out the importance of considering service users' views on a particular service as their needs and opinions may be quite different from those which service providers might expect. Given this, Bucknall ${ }^{9}$ suggests that the appropriate place for consumer opinion surveys is 'as an indicator of which aspects of the service might be changed to improve clients' responses'.

The aims of the present study are threefold:

1 To assess whether the aims of Goal Planning as outlined by Kennedy and Pearce ${ }^{6}$ have been met at QUENSIC. Specifically, whether patients considered the Goal Planning approach to have been helpful in providing information about their condition, whether they felt it increased their sense of control over their rehabilitation and whether there were any areas of their rehabilitation they felt goal planning did not cover.

2 To investigate the relationship between information provision and control with the aim of clarifying those factors which foster participation in rehabilitation.

3 To assess staff perceptions of Goal Planning in terms of its usefulness, its impact on their workload and their ideas about areas where their training, or the system as a whole, might be improved.

\section{Methods}

\section{Subjects}

Patient Group The sample consisted of 33 patients with spinal cord injuries who participated in Goal Planning during their hospital admission. Of these patients, $27(81.8 \%)$ were male and six female and the age range was from 16 to 72 years (mean age of 38.6 years). Patients came from a fairly representative cross section of regions within Scotland, with the majority $(42.4 \%)$ from the Strathclyde region. Figure 1 shows the distribution of the level of injury of the patients.
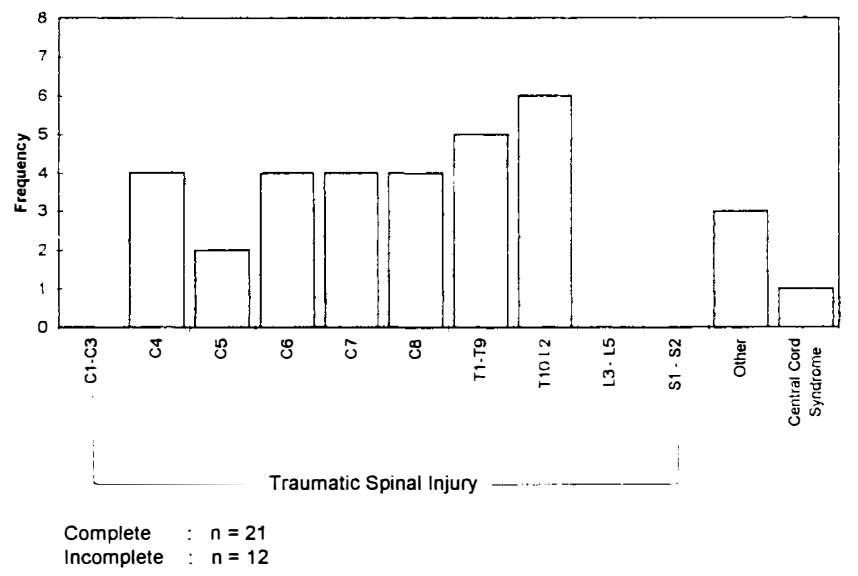

Figure 1 Distribution of Level of Patients
Fourteen $(42.4 \%)$ patients had an injury at C7 or above. One patient had a central cord syndrome and, of the three patients represented on the graph in the 'other' category, one had spinal tuberculosis, one had a neoplasm and the third had 'Locked in' syndrome. The modal injury category was between T10 and L2. Almost two thirds (21) of these injuries were complete. Two thirds (22) of the patients who had participated in Goal Planning had been discharged by the end of the assessment period. Of these, the mean length of admission was 21.3 weeks (range $=7-37$ ). The first Goal Planning meeting was held on average 7.1 weeks (range $=2-18$ weeks) after admission.

Staff/keyworker group A total of 13 staff members participated in the study, nine $(69.2 \%)$ of whom were female. Seven $(53.8 \%)$ were nursing staff and the remaining six were split equally between physiotherapy and occupational therapy.

\section{Measures}

Patient and staff views were elicted using specially constructed questionnaires (see Appendix 1).

\section{Procedure}

Prior to the introduction of Goal Planning, the Centre's multidisciplinary staff team all underwent keyworker training directed by the Centre's psychologist (first author) and using a training package based on that of Kennedy. ${ }^{5}$

Upon admission to the Centre, patients were allocated a member of staff to be their keyworker. The keyworker is responsible for completing an intitial needs assessment* and organises and facilitates subsequent Goal Planning meetings as well as acting as an advocate for the patient where necessary. The frequency of Goal Planning meetings varied from weekly to monthly according to individual needs and circumstances and was at the discretion of the therapists involved. However, the average frequency of meetings was fortnightly. Goal planning finished when the rehabilitation targets identified as a result of the original needs assessment were achieved. The needs assessment was again completed at this point as a safety check to ensure that nothing had been missed. Although at this point, the patient was deemed to have completed rehabilitation, discharge was not always possible for a variety of practical reasons such as delays in re-housing and housing adaption.

Towards the end of Goal Planning, the psychologist administered questionnaires to patients and assisted those who were unable to complete them. The patients for whom the psychologist had been keyworker $(n=4)$

*The Needs assessment covers 14 key areas in SCI rehabilitation and was based on a similar document used in Stoke Mandeville National Spinal Injury Centre and adapted for use with patients in QUENSIC. The schedule used can be obtained from the first author. 
were omitted from the final sample as they may have felt particular pressure to give socially desirable responses.

In addition to the information obtained from the patient questionnaires, demographic details were obtained from a detailed review of case notes.

Keyworkers also completed questionnaires assessing their views on the Goal Planning process once Goal Planning had been completed.

\section{Results}

\section{Patients' responses}

Perceptions of goal planning: Patient ratings of informativeness All patients reported being aware that a Goal Planning programme was being used in their rehabilitation. Figure 2 illustrates patients, ratings of how helpful they found Goal Planning to be in providing information about their condition. As we can see, $36.5 \%$ (7) people found it to be 'extremely helpful' in this respect. The modal response on the scale from 1 ('not at all helpful') to 7 ('extremely helpful') was 5 , indicating that patients generally seemed to find Goal Planning to be informative about their condition.

When relatives or friends were involved in the Goal Planning process (i.e. they attended Goal Planning meetings regularly), they were requested to indicate how helpful they had found Goal Planning to be in helping them understand rehabilitation. In just over half of cases (18), relatives or friends completed this part of the questionnaire. Of these eight $(44.4 \%)$ relatives/friends indicated that they had found Goal Planning to be 'extremely helpful' in this respect, with the remainder of responses falling within the 'helpful' half of the rating scale.

Patient ratings of control over rehabilitation Figure 3 illustrates patient perceptions of control over planning their rehabilitation. The majority of patients $(60.6 \%)$ felt they had 'a fair amount of control', while six patients $(18.2 \%)$ felt Goal Planning offered 'complete

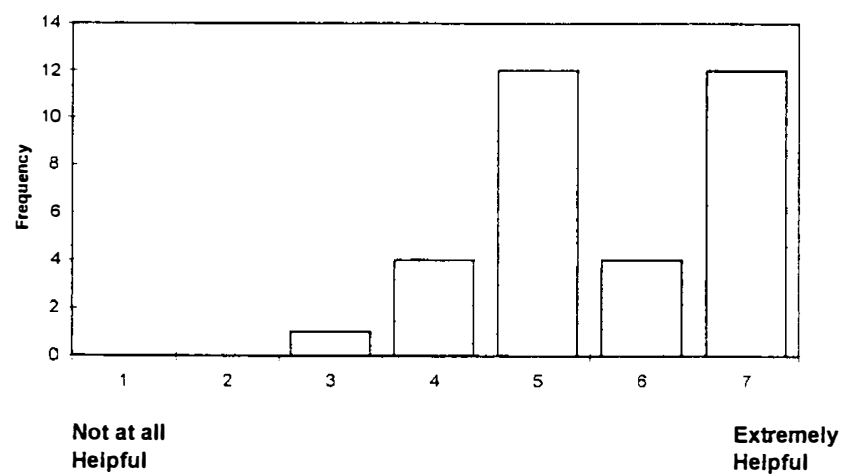

Figure 2 Patients' ratings of how helpful they found Goal Planning to be providing information control'. One patient felt it offered no control.

The basic premise of the Goal Planning approach is that by increasing patient information about their condition and rehabilitation, perceived control is improved and thereby rehabilitation is facilitated. As the data in question is ordinal scaled, it necessitated the use of non-parametric correlation and Kendall's Tau B was chosen on the basis that there was likely to be a substantial number of tied ranks. ${ }^{10}$ A Kendall's Tau B correlation produced a correlation coefficient of $0.44(P<0.002)$ indicating that there is a significant relationship between the provision of information and control over rehabilitation.

Kendall's Tau B correlations were also carried out in order to assess whether perceptions of informativeness or control were associated with the level of injury. Correlation coefficients of 0.06 and 0.09 were obtained between level of injury and informativeness and control, respectively, indicating that there were no significant associations (at $P<0.05$ level of significance) between either of these two variables and level of injury.

Mann - Whitney tests were used to compare ratings of informativeness and control for those with complete and those with incomplete injuries. In terms of perceived informativeness, mean ranks of 17.10 and 16.83 were found for those with complete $(n=21)$ and incomplete $(n=12)$ injuries, respectively (high scores indicative of high perceived informativeness): $U=124.0, W=202.0$, exact two-tailed probability $P=0.9559$. Thus, those with complete and those with incomplete injuries did not appear to differ significantly in their perceptions of how informative they found Goal Planning to be concerning their injury.

Neither were there significant differences found in perceived control over rehabilitation for individuals with complete and incomplete injuries: MannWhitney tests gave mean ranks of 18.57 and 14.25 for those with complete and those with incomplete injuries respectively (a high score is indicative of high perceived control): $U=93.0, W=171.0$, exact twotailed probability $-P=0.2276$.

As length of admission was determined by factors

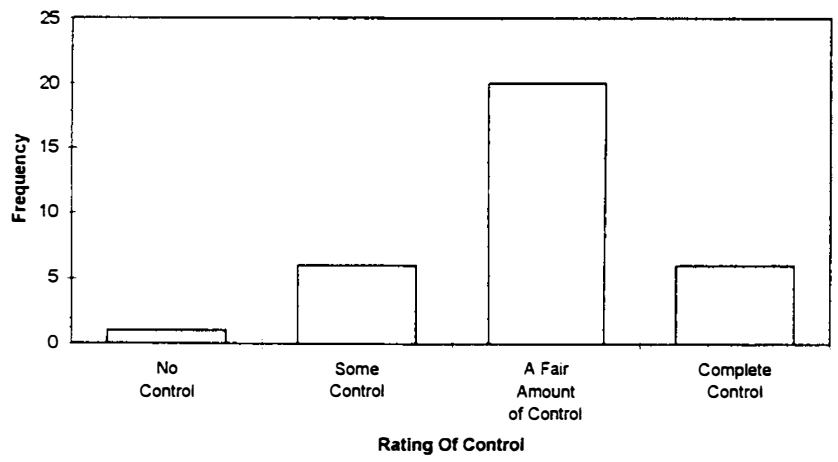

Figure 3 Patients' perceptions of control over planning their rehabilitation 
other than the efficiency or otherwise of rehabilitation (eg housing issues), this relationship was not considered.

Qualitative information was also sought concerning patients' views on any areas they felt their rehabilitation programme did not cover. Four patients $(12 \%)$ reported feeling that there were areas of rehabilitation which Goal Planning did not cover. Answers provided were disparate and included 'medical problems', 'sexual issues', 'paralysis', 'sweating under arms' and 'payment for bed'. No two patients mentioned any one of these areas.

\section{Keyworker responses}

Twelve keyworkers $(92.3 \%)$ reported feeling that Goal Planning was 'useful in every case' in spinal injuries management, and the remaining keyworker indicated that they felt it to be 'sometimes useful'. All keyworkers responded that they felt Goal Planning to be beneficial to patients, staff and relatives.

It was felt that an important factor in determining staff satisfaction with a new form of intervention might be their beliefs about how it impinged on their workload. As Figure 4 illustrates, two keyworkers $(15.4 \%)$ reported feeling that it made their workload 'easier', five $(38.5 \%)$ that it made 'no difference' and six $(46.1 \%)$ that it made their workload 'harder'. However, of the latter six keyworkers, four specifically commented that Goal Planning did not make their workload 'harder' per se, but that it tended to be time consuming.

More qualitative information was obtained by asking keyworkers open ended questions concerning their views on how training and the system as a whole, might be improved. Six keyworkers (46.2\%) maintained that the training did not require alteration. Of the seven keyworkers who suggested changes, two proposed the use of role-play and individual keyworkers made the following suggestions: the introduction of training 'up-date' sessions, more teaching on negotiation and counselling skills, the provision of more practical information about programme implementation, the introduction of a mentorship scheme, and initially, being allowed to sit in on Goal Planning meetings without being expected to contribute.

Concerning more general system changes, $69.2 \%$ of keyworkers (9) made no suggestions or commented

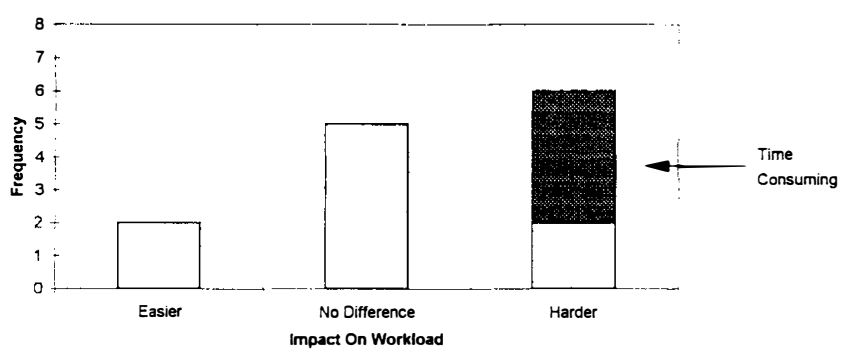

Figure 4 Keyworkers' perceptions of impact of goal planning on workload that they felt the existing system was adequate as it stood. Individual suggestions included earlier involvement with community services, more regular input from medical staff, the involvement of an associate worker in addition to the main keyworker and attempts to try to alleviate a burgeoning case load.

When keyworkers were given the opportunity to try to sum up their thoughts on the use of Goal Planning in spinal cord injury rehabilitation, their responses revealed a number of themes. Almost half (46.2\%) commented that Goal Planning increases patient knowledge about spinal cord injury and $38.5 \%$ of keyworkers felt their own knowledge had been improved. Other positive aspects of Goal Planning mentioned included increased patient control over rehabilitation, the provision of a forum for discussion, and the fact that Goal Planning directed patient care. In addition, $15.4 \%$ of keyworkers believed that Goal Planning increased staff accountability. No negative comments were made.

\section{Discussion}

It would appear from patients' responses that the Goal Planning programme implemented at QUENSIC provided patients with helpful information on their condition and that this was associated with patients feeling that they had control over their rehabilitation. When relatives or friends were involved in the Goal Planning process, they also seemed to find the programme useful in understanding rehabilitation. Although previous research ${ }^{11}$ has shown differences between those with complete and incomplete lesions in terms of their adjustment to their condition, the present study found no significant differences between these two groups on either perceived informativeness or control. Neither was there a significant association between level of spinal injury and perceptions of informativeness or control.

The results relating to keyworker perceptions of Goal Planning, although mostly qualitative in nature, suggest that they tend to view it as a useful intervention of benefit to patients, relatives and staff. Goal Planning was believed to cause no extra work in many cases, although a number of keyworkers felt that it was a time-consuming form of rehabilitation. It is possible that this is a reflection of the fact that the programme represents a new and different way of working which may become less time consuming and labour intensive as staff become more accustomed to it. Training was generally felt to be adequate, although suggestions for the use of role play and a more gradual assumption of responsibility were suggested, ie the introduction of a mentorship scheme, and being allowed to sit in on Goal Planning meetings prior to participating in them. Many of these suggestions are currently being adopted in the training of new keyworkers. More generally, keyworkers commented that they felt the existing system would operate more effectively given earlier involvement with community 
services, more regular input from medical staff, and the involvement of as associate worker to ease individual workload. These comments validated existing concerns within QUENSIC.

A number of methodological limitations inherent in the present study may be worth considering when evaluating the results and attempting to draw conclusions. Social desirability is a problem inherent in customer satisfaction evaluations and may be pertinent here. Although efforts were taken to minimise 'pressure to please' by omitting those patients for whom the psychologist administering the questionnaires was keyworker, it is possible that patients were aware of the key role the psychologist had in the training and overseeing of the Goal Planning programme. Also, as McPhee et al ${ }^{12}$ point out, it is inadequate to ask if patients are 'satisfied' in general terms and this criticism may be levied here: it might have been helpful not only to ask 'how informative' or 'how much control', but 'what' was the most informative or helpful or provided the most control and to provide patients with checklists.

Nevertheless, the results of this study suggest that patients found Goal Planning to be informative and that this was associated with patients feeling that they had control over their rehabilitation. It was not possible to conclude whether the implementation of Goal Planning necessarily resulted in shorter admissions or a decreased rate of re-admission as various medical and social factors confound the situation, eg often inadequate housing arrangements were the reason for discharge delays, despite early liaison with community agencies.

Partridge and Johnston ${ }^{3}$ have highlighted the importance of other potential mediating variables which may help explain the variance in recovery from physical disability, including individual differences in coping style. Rotter ${ }^{13,14}$ distinguished between those with internal locus of control (ie believe that rewards are contingent upon their own behaviour) from those with an external locus of control (ie believe that positive or negative events in their lives are unrelated to their behaviour and determined by forces outside of themselves). Subsequent work using this construct $^{11,15}$ has shown that amongst people with spinal injuries, an internal locus of control is predictive of better psychological adjustment and better functional outcomes.

However, Strickland ${ }^{16}$ suggests that health care approaches are most effective when tailored to an individuals' expectations. Therefore, one might expect that internal patients will tend to respond best to a regime promoting personal autonomy, choice and decision making (like Goal Planning), whereas more external patients may respond optimally to a more structured and controlled treatment environment. Thus it seems that choice and perceived control may not be beneficial per se, but perhaps only when 'meshed' with an internal coping style.

Morrison, Johnston and MacWalter ${ }^{17}$ have also highlighted the importance of self-efficacy beliefs in determining rehabilitation outcomes. Self efficacy beliefs refer to a person's perceived ability to successfully carry out a given response. Thus, it is possible for a person to have an internal locus of control, ie believe that what they do is important for their recovery, but may feel unable to successfully perform the necessary actions to take that control. It would therefore seem important for future research to ascertain whether rehabilitation efforts such as Goal Planning foster greater internality and enhanced selfefficacy beliefs and to investigate the effects of these variables on psychological and behavioural outcomes.

\section{Conclusions}

Given the limitations of social desirability and the inherent statistical restrictions, this study provides a preliminary attempt at canvassing both patient and staff views on the first year of Goal Plannning within the area of spinal injuries rehabilitation. It represents an attempt to evaluate customer satisfaction with the programme and to assess the mechanism by which improvements might be gained. The results have indicated that patients generally feel Goal Planning to be informative about their injury and helpful in promoting a sense of control over rehabilitation, and also suggests that these two factors may be linked. In addition, staff appear to have favourable views on the benefits of Goal Planning for patients, relatives and staff, although they acknowledge the impact it has on their workload.

\section{References}

I The Health of the Nation: A strategy for Health in England. Presented to Parliament by the Secretary of State for Health (July 1992) London: HMSO.

2 The Patient's Charter: A Charter for Health. Produced by the Scottish Office (Sept. 1991) Edinburgh: HMSO.

3 Partridge C, Johnston M. Perceived control of recovery from physical disability: measurement and prediction. British Journal of Clinical Psychology 1989; 28: $53-59$.

4 Norris-Baker C, Stephens M, Rintala D. Patient behaviour as a predictor of outcome in spinal cord inury. Archives of Phisiological and Medical Research 1981; 62: 602-608.

5 Kennedy P, Walker L, White D. Ecological evaluation of goal planning and advocacy in rehabilitative environment for spinal cord injured people. Paraplegia 1991; 29: 197-202.

6 Kennedy P, Pearce N. Goal planning, needs assessment and advocacy. Health Services Management 1993; March: 17-19.

7 Houts P. Scott R. Goal Planning with Developmentally Disabled Persons: Procedures for Developing. In: Individualised Client Plan. Milton Hershey Centre: Pennsylvania, 1975.

8 Trinder H, Mitchell SA, Newness C, Todd LMK. Valuing patient feedback II: Shelton Hospital's rehabilitation wards. Clinical Psychology Forum 1994; 74: 21 - 25.

9 Bucknall A. Evaluation of a client satisfaction questionnaire. Clinical Psychology Forum 1994; 63: 22 - 26.

10 Ferguson GA. Statistical Analysis in Psychology and Education 3rd ed. London: McGraw Hill, 1971. 
11 Stuckey N. Pilot study to examine the adjustment, emotional state, locus of control and social support of spinally injured patients. Final report to Disability Research Committee, Scottish Home and Health Department, Edinburgh (Grant no. D/RED/6/ 31/2/F2), 1993.

12 McPhee CB, Zusman J, Ross RH. Measurement of patient satisfaction. A survey of practices in Community Mental Health Centres. Comprehensive Psychiatry 1975; 16: 399-404.

13 Rotter JB. Generalised expectancies for internal versus external locus of control of reinforcement. Psychological Monographs 1966; 80 (1): whole no. 609.

14 Rotter JB. The Development and Application of Social Learning Theory. New York: Praeger, 1982.

Appendix 1 (a) Patient Questionnaires

\section{Goal Planning}

1. Were you aware that Goal Planning was being used in your rehabilitation? Please circle:

$$
\text { Yes No }
$$

2. Is Goal Planning helpful in giving you information about your condition? Please rate by circling a number from 1 to 7 , where 1 is "not at all helpful" and 7 is "extremely helpful".

Not at all helpful

Extremely helpful

$\begin{array}{lllllll}1 & 2 & 3 & 4 & 5 & 6 & 7\end{array}$

3. Were there areas of your rehabilitation that Goal Planning did not help with.

\section{Yes No}

If yes, then please describe below which areas you would most like more help with.

4. Your relatives, family or friends may have been involved in Goal Planning meetings. Please could you ask them to rate how helpful they think the meetings been in helping them understand your rehabilitation. Please ask them to rate by circling a number from 1 to 7 , where 1 is "not at all helpful" and 7 is "extremely helpful".

Not at all helpful

$\begin{array}{lllllll}1 & 2 & 3 & 4 & 5 & 6 & 7\end{array}$

5. How much control do you feel you have in planning your rehabilitation? Please circle:

no control

some control

a fair amount of control

complete control
Extremely helpful

$$
7
$$

15 Shulz R, Decker A. Long-term adjustment to physical disability: the role of social support, perceived control and self-blame. Journal of Personality and Social Psychology 1985; 48: 11621172 .

16 Strickland BR. Internal external expectancies and health related behaviours. Journal of Consulting and Clinical Psychology 1978; 46 (6): $1192-1211$.

17 Morrison V, Johnston M, MacWalter R. Disability following stroke: Psychological predictors of residual disability. Final report to the Chest, Heart and Stroke Association (Scotland), 1994.

Appendix 1 (b) Keyworker questionnaires

\section{Goal Planning Evaluation}

1. How would you rate the use of Goal Planning in spinal injuries management? Please circle:

no use

sometimes useful

useful in every case

indespensable

2. Does being a keyworker make your workload:

easier

harder

no difference

3. Do you think Goal Planning is of benefit to:

$\begin{array}{ll}\text { Patients } & \text { Yes/No } \\ \text { Staff } & \text { Yes/No } \\ \text { Relatives } & \text { Yes/No }\end{array}$

4. Are there changes you would like to make in the keyworker training?

Please describe below

5. Are there changes you would like to make in the way the system is implemented?

Please descibe below

6. Please sum up your thoughts in general on the use of the Goal Planning system in spinal injuries rehabilitation. 